\title{
Unitarity constraints on anomalous quartic couplings
}

\author{
Eduardo da Silva Almeida $\odot^{*}$ and O. J. P. Éboli® ${ }^{\dagger}$ \\ Instituto de Física, Universidade de São Paulo, São Paulo-SP, 05508-090, Brazil \\ M. C. Gonzalez-Garcia \\ Institució Catalana de Recerca i Estudis Avançats (ICREA), \\ Departament d'Estructura i Constituents de la Matèria, Universitat de Barcelona, \\ 647 Diagonal, E-08028 Barcelona, Spain \\ and C.N. Yang Institute for Theoretical Physics, SUNY at Stony Brook, \\ Stony Brook, New York 11794-3840, USA
}

(Received 21 April 2020; accepted 4 June 2020; published 26 June 2020)

\begin{abstract}
We obtain the partial-wave unitarity constraints on the lowest-dimension effective operators which generate anomalous quartic gauge couplings but leave the triple gauge couplings unaffected. We consider operator expansions with linear and nonlinear realizations of the electroweak symmetry and explore the multidimensional parameter space of the coefficients of the relevant operators: 18 dimension-eight operators in the linear expansion and $5 \mathcal{O}\left(p^{4}\right)$ operators in the derivative expansion. We study two-to-two scattering of electroweak gauge bosons and Higgs bosons, taking into account all coupled channels and all possible helicity amplitudes for the $J=0,1$ partial waves. In general, the bounds degrade by factors of a few when several operator coefficients are considered to be nonvanishing simultaneously. However, this requires considering constraints from both $J=0$ and $J=1$ partial waves for some sets of operators.
\end{abstract}

DOI: 10.1103/PhysRevD.101.113003

\section{INTRODUCTION}

The structure of the triple (TGC) and quartic (QGC) electroweak gauge-boson interactions in the Standard Model (SM) is determined by the gauge symmetry $S U(2)_{L} \otimes U(1)_{Y}$. Therefore, it is important to measure both TGC and QGC, not only to further test the SM or have indications of new physics, but also to determine whether the gauge symmetry is realized linearly or nonlinearly in the low-energy effective theory of the electroweak symmetry breaking sector [1].

Generically, deviations from the SM predictions for TGC and QGC are generated by higher-order operators parametrizing indirect effects of new physics. Collider experiments probe TGC in the pair production of electroweak gauge bosons, while the study of QGC requires the production of three electroweak vector bosons, the exclusive production of gauge-boson pairs, or the vector-bosonscattering production of electroweak vector boson pairs [2-17]. Therefore, the Wilson coefficients of effective

\footnotetext{
*eduardo.silva.almeida@usp.br †eboli@if.usp.br

*concha@insti.physics.sunysb.edu
}

Published by the American Physical Society under the terms of the Creative Commons Attribution 4.0 International license. Further distribution of this work must maintain attribution to the author(s) and the published article's title, journal citation, and DOI. Funded by SCOAP. operators that contain both TGC and QGC are more strongly constrained through the study of their TGC component.

For this reason, most of the present LHC searches for effects of QGC focus on the so-called genuine QGC operators, that is, operators that generate QGC but do not have any TGC associated with them. In a scenario where the $S U(2)_{L} \otimes U(1)_{Y}$ is realized linearly, the lowestorder QGC are given by dimension-eight operators [18]. Alternatively, if the gauge symmetry is implemented nonlinearly, the lowest-order QGC appear at $\mathcal{O}\left(p^{4}\right)[19,20]$.

It is well known that departures of the TGC and QGC from the SM predictions lead to the growth of scattering amplitudes [21], signalizing the existence of new physics. Thus, when probing anomalous QGC, one must verify whether perturbative partial-wave unitarity is satisfied to guarantee consistency of the analyses. This is all well established, and it has been previously addressed in the literature [4,22-28]. It is also implemented in some form in the QGC searches by both the ATLAS and CMS collaborations (see for instance Refs. [9-13,15,17]), either by introducing $a d$ hoc form factors or unitarization procedures (see Ref. [29] for a study of the dependence on the unitarization procedure employed), or by directly evaluating the maximum center-of-mass energy allowed by unitarity as obtained from the VBFNLO framework [25]. However, these unitarity studies are not complete since they consider just a few scattering channels or a limited set of QGC effective operators, or they restricted the analysis to the $J=0$ partial wave. 
In this work we complement the existing literature on the subject by systematically presenting the unitarity bounds in the multidimensional parameter space of the coefficients of the relevant operators in both linear and nonlinear realizations of the electroweak symmetry. We study two-to-two scattering of electroweak gauge bosons and Higgs bosons, taking into account all coupled channels and all possible helicity amplitudes for the $J=0,1$ partial waves. Indeed, we find that $J=1$ partial-wave unitarity effects are relevant to derive the most stringent limits in some scenarios when the effects of several operators are considered simultaneously.

This paper is organized as follows: We present in Sec. II the QCG operators that we consider in our analyses, as well as basic expressions of partial-wave unitarity needed for our studies. Section III contains our results, which are discussed in Sec. IV.

\section{ANALYSES FRAMEWORK}

Here, we introduce the effective interactions considered in this work, as well as the unitarity relations that we use to constrain them.

\section{A. Effective Lagrangian}

\section{Linear realization of the gauge symmetry}

Assuming that the new state observed in 2012 is in fact the SM Higgs boson and that it belongs to an electroweak scalar doublet, we can construct a low-energy effective theory where the $S U(2)_{L} \otimes U(1)_{Y}$ gauge symmetry is linearly realized [30-36], which takes the form

$$
\mathcal{L}_{\text {eff }}=\mathcal{L}_{\mathrm{SM}}+\sum_{n=5}^{\infty} \sum_{i} \frac{f_{i}^{(n)}}{\Lambda^{n-4}} \mathcal{O}_{i}^{(n)},
$$

where the dimension- $n$ operators $\mathcal{O}_{i}^{(n)}$ involve gauge bosons, Higgs doublets, fermionic fields, and covariant derivatives of these fields. Each operator has a corresponding Wilson coefficient $f_{i}^{(n)}$, and $\Lambda$ is the characteristic energy scale at which new physics (NP) becomes apparent.

Here, we are interested in operators that lead to QGC without a TGC counterpart. The lowest dimension of such genuine QGC operators is eight [18]. In what follows, we consider the bosonic dimension-eight operators relevant to two-to-two scattering processes involving Higgs and/or gauge bosons at tree level, and that conserve $C$ and $P$ [37]. Moreover, we classify them by the number of gauge-boson strength fields contained in the operator.

In the first class of genuine QGC, the operators contain just covariant derivatives of the Higgs field:

$$
\begin{aligned}
\mathcal{O}_{S, 0} & =\left[\left(D_{\mu} \Phi\right)^{\dagger} D_{\nu} \Phi\right] \times\left[\left(D^{\mu} \Phi\right)^{\dagger} D^{\nu} \Phi\right], \\
\mathcal{O}_{S, 1} & =\left[\left(D_{\mu} \Phi\right)^{\dagger} D^{\mu} \Phi\right] \times\left[\left(D_{\nu} \Phi\right)^{\dagger} D^{\nu} \Phi\right], \\
\mathcal{O}_{S, 2} & =\left[\left(D_{\mu} \Phi\right)^{\dagger} D_{\nu} \Phi\right] \times\left[\left(D^{\nu} \Phi\right)^{\dagger} D^{\mu} \Phi\right],
\end{aligned}
$$

where $\Phi$ stands for the Higgs doublet, the covariant derivative is given by $D_{\mu} \Phi=\left(\partial_{\mu}+i g W_{\mu}^{j} \frac{\sigma^{j}}{2}+i g^{\prime} B_{\mu} \frac{1}{2}\right) \Phi$, and $\sigma^{j}(j=1,2,3)$ represent the Pauli matrices.

In the second class of genuine QGC, the operators exhibit two covariant derivatives of the Higgs field, as well as two field strengths:

$$
\begin{aligned}
\mathcal{O}_{M, 0} & =\operatorname{Tr}\left[\hat{W}_{\mu \nu} \hat{W}^{\mu \nu}\right] \times\left[\left(D_{\beta} \Phi\right)^{\dagger} D^{\beta} \Phi\right], \\
\mathcal{O}_{M, 1} & =\operatorname{Tr}\left[\hat{W}_{\mu \nu} \hat{W}^{\nu \beta}\right] \times\left[\left(D_{\beta} \Phi\right)^{\dagger} D^{\mu} \Phi\right], \\
\mathcal{O}_{M, 2} & =\left[B_{\mu \nu} B^{\mu \nu}\right] \times\left[\left(D_{\beta} \Phi\right)^{\dagger} D^{\beta} \Phi\right], \\
\mathcal{O}_{M, 3} & =\left[B_{\mu \nu} B^{\nu \beta}\right] \times\left[\left(D_{\beta} \Phi\right)^{\dagger} D^{\mu} \Phi\right], \\
\mathcal{O}_{M, 4} & =\left[\left(D_{\mu} \Phi\right)^{\dagger} \hat{W}_{\beta \nu} D^{\mu} \Phi\right] \times B^{\beta \nu}, \\
\mathcal{O}_{M, 5} & =\left[\left(D_{\mu} \Phi\right)^{\dagger} \hat{W}_{\beta \nu} D^{\nu} \Phi\right] \times B^{\beta \mu}+\text { H.c. }, \\
\mathcal{O}_{M, 7} & =\left[\left(D_{\mu} \Phi\right)^{\dagger} \hat{W}_{\beta \nu} \hat{W}^{\beta \mu} D^{\nu} \Phi\right] .
\end{aligned}
$$

where $\hat{W}_{\mu \nu} \equiv W_{\mu \nu}^{j} \frac{\sigma^{j}}{2}$ is the $S U(2)_{L}$ field strength while $B_{\mu \nu}$ stands for the $U(1)_{Y}$ one.

In addition to the above operators, there are also genuine QGC ones that contain just field strengths:

$\mathcal{O}_{T, 0}=\operatorname{Tr}\left[\hat{W}_{\mu \nu} \hat{W}^{\mu \nu}\right] \times \operatorname{Tr}\left[\hat{W}_{\alpha \beta} \hat{W}^{\alpha \beta}\right]$,

$\mathcal{O}_{T, 1}=\operatorname{Tr}\left[\hat{W}_{\alpha \nu} \hat{W}^{\mu \beta}\right] \times \operatorname{Tr}\left[\hat{W}_{\mu \beta} \hat{W}^{\alpha \nu}\right]$

$\mathcal{O}_{T, 2}=\operatorname{Tr}\left[\hat{W}_{\alpha \mu} \hat{W}^{\mu \beta}\right] \times \operatorname{Tr}\left[\hat{W}_{\beta \nu} \hat{W}^{\nu \alpha}\right]$,

$\mathcal{O}_{T, 5}=\operatorname{Tr}\left[\hat{W}_{\mu \nu} \hat{W}^{\mu \nu}\right] \times B_{\alpha \beta} B^{\alpha \beta}$

$\mathcal{O}_{T, 6}=\operatorname{Tr}\left[\hat{W}_{\alpha \nu} \hat{W}^{\mu \beta}\right] \times B_{\mu \beta} B^{\alpha \nu}$,

$\mathcal{O}_{T, 7}=\operatorname{Tr}\left[\hat{W}_{\alpha \mu} \hat{W}^{\mu \beta}\right] \times B_{\beta \nu} B^{\nu \alpha}$

$\mathcal{O}_{T, 8}=B_{\mu \nu} B^{\mu \nu} B_{\alpha \beta} B^{\alpha \beta}, \quad \mathcal{O}_{T, 9}=B_{\alpha \mu} B^{\mu \beta} B_{\beta \nu} B^{\nu \alpha}$.

These 18 operators induce all possible modifications to vertices $V V V V, V V V H$, and $V V H H\left(V=W^{ \pm}, Z\right.$ and $\left.A\right)$ that are compatible with electric charge, $C$ and $P$ conservation; for further details on the anomalous vertices generated by each dimension-eight operator, see Ref. [37].

\section{Nonlinear $\mathcal{O}\left(p^{4}\right)$ realization of the gauge symmetry}

In dynamical scenarios, the Higgs boson is a composite state; i.e., it is a pseudo-Nambu-Goldstone boson of an exact global symmetry. Therefore, the gauge symmetry of the low-energy effective Lagrangian is realized nonlinearly [38-41], and the effective Lagrangian is a derivative expansion. In this case, the effective Lagrangian is written in terms of the SM fermions and gauge bosons and of the physical Higgs $h$. The building block at low energies is a 
dimensionless unitary matrix transforming as a bidoublet of the global symmetry $S U(2)_{L} \otimes S U(2)_{R}$ :

$$
\mathbf{U}(x)=e^{i \sigma_{a} \pi^{a}(x) / v}, \quad \mathbf{U}(x) \rightarrow L \mathbf{U}(x) R^{\dagger},
$$

where $L, R$ denote $S U(2)_{L, R}$ global transformations, respectively, and $\pi^{a}$ are the Goldstone bosons. Its covariant derivative is given by

$\mathbf{D}_{\mu} \mathbf{U}(x) \equiv \partial_{\mu} \mathbf{U}(x)+i g \frac{\sigma^{j}}{2} W_{\mu}^{i}(x) \mathbf{U}(x)-\frac{i g^{\prime}}{2} B_{\mu}(x) \mathbf{U}(x) \sigma_{3}$.

From this basic element, it is possible to construct the vector chiral field

$$
V_{\mu} \equiv\left(\mathbf{D}_{\mu} \mathbf{U}\right) \mathbf{U}^{\dagger}
$$

and the scalar chiral field $T \equiv \mathbf{U} \sigma_{3} \mathbf{U}^{\dagger}$. For further details see Ref. [37].

The lowest operators respecting $C$ and $P$ and exhibiting genuine QGC are of order $p^{4}$, which, in the notation of Refs. $[19,20]$, are

$$
\begin{aligned}
\mathcal{P}_{6} & =\operatorname{Tr}\left[V^{\mu} V_{\mu}\right] \operatorname{Tr}\left[V^{\nu} V_{\nu}\right] \mathcal{F}_{6}(h), \\
\mathcal{P}_{11} & =\operatorname{Tr}\left[V^{\mu} V^{\nu}\right] \operatorname{Tr}\left[V_{\mu} V_{\nu}\right] \mathcal{F}_{11}(h),
\end{aligned}
$$

which respect the $S U(2)_{c}$ custodial symmetry and

$$
\begin{aligned}
& \mathcal{P}_{23}=\operatorname{Tr}\left[V^{\mu} V_{\mu}\right]\left(\operatorname{Tr}\left[T V_{\nu}\right]\right)^{2} \mathcal{F}_{23}(h), \\
& \mathcal{P}_{24}=\operatorname{Tr}\left[V^{\mu} V^{\nu}\right] \operatorname{Tr}\left[T V_{\mu}\right] \operatorname{Tr}\left[T V_{\nu}\right] \mathcal{F}_{24}(h) \\
& \mathcal{P}_{26}=\left(\operatorname{Tr}\left[T V_{\mu}\right] \operatorname{Tr}\left[T V_{\nu}\right]\right)^{2} \mathcal{F}_{26}(h),
\end{aligned}
$$

which violate $S U(2)_{c}$. Note that $\mathcal{F}_{i}(h)$ are generic functions parametrizing the chiral-symmetry breaking interactions of $h$. As we are looking for operators whose lowest order vertex contains four gauge bosons, we take $\mathcal{F}_{i}=1$. So, the most general Lagrangian at $\mathcal{O}\left(p^{4}\right)$ for genuine QGC is

$$
\mathcal{L}_{\mathrm{QGC}}^{p=4}=\sum_{i=6,11,23,24,26} f_{P, i} \mathcal{P}_{i}
$$

It is interesting to notice that the above nonlinear operators do not contain photons.

\section{B. Partial-wave unitarity}

In the two-to-two scattering of electroweak gauge bosons $(V)$,

$$
V_{1 \lambda_{1}} V_{2 \lambda_{2}} \rightarrow V_{3 \lambda_{3}} V_{4 \lambda_{4}}
$$

the corresponding helicity amplitude can be expanded in partial waves in the center-of-mass system as [42]

$$
\begin{aligned}
\mathcal{M}( & \left.V_{1 \lambda_{1}} V_{2 \lambda_{2}} \rightarrow V_{3 \lambda_{3}} V_{4 \lambda_{4}}\right) \\
= & 16 \pi \sum_{J}(2 J+1) \sqrt{1+\delta_{V_{1 \lambda_{1}}}^{V_{2 \lambda_{2}}}} \sqrt{1+\delta_{V_{3 \lambda_{3}}}^{V_{4 \lambda_{4}}}} d_{\lambda \mu}^{J}(\theta) \\
& \times e^{i M \varphi} T^{J}\left(V_{1 \lambda_{1}} V_{2 \lambda_{2}} \rightarrow V_{3 \lambda_{3}} V_{4 \lambda_{4}}\right),
\end{aligned}
$$

where $\lambda=\lambda_{1}-\lambda_{2}, \mu=\lambda_{3}-\lambda_{4}, \quad M=\lambda_{1}-\lambda_{2}-\lambda_{3}+\lambda_{4}$, and $\theta(\varphi)$ is the polar (azimuth) scattering angle. Here, $d$ is the usual Wigner rotation matrix. For processes where we substitute a vector boson by a Higgs, this expression can be used by setting the corresponding $\lambda$ to zero.

Partial-wave unitarity for a given elastic channel requires that

$$
\left|T^{J}\left(V_{1 \lambda_{1}} V_{2 \lambda_{2}} \rightarrow V_{1 \lambda_{1}} V_{2 \lambda_{2}}\right)\right| \leq 1
$$

where we considered the limit $s \gg\left(M_{V_{1}}+M_{V_{2}}\right)^{2}$. More stringent bounds can be obtained by diagonalizing $T^{J}$ in the particle and helicity space and then applying the condition in Eq. (13) to each of the eigenvalues.

In our analysis we evaluated $T^{0}$ and $T^{1}$ amplitude matrices in particle and parameter space as a function of the Wilson coefficients of the dimension-eight operators and the nonlinear ones. These matrices are formed with the $s$-divergent parts of the amplitudes corresponding to all combinations of gauge boson and Higgs pairs with a given total charge $Q=2,1,0$ with possible projections on a given partial wave $J$, which are

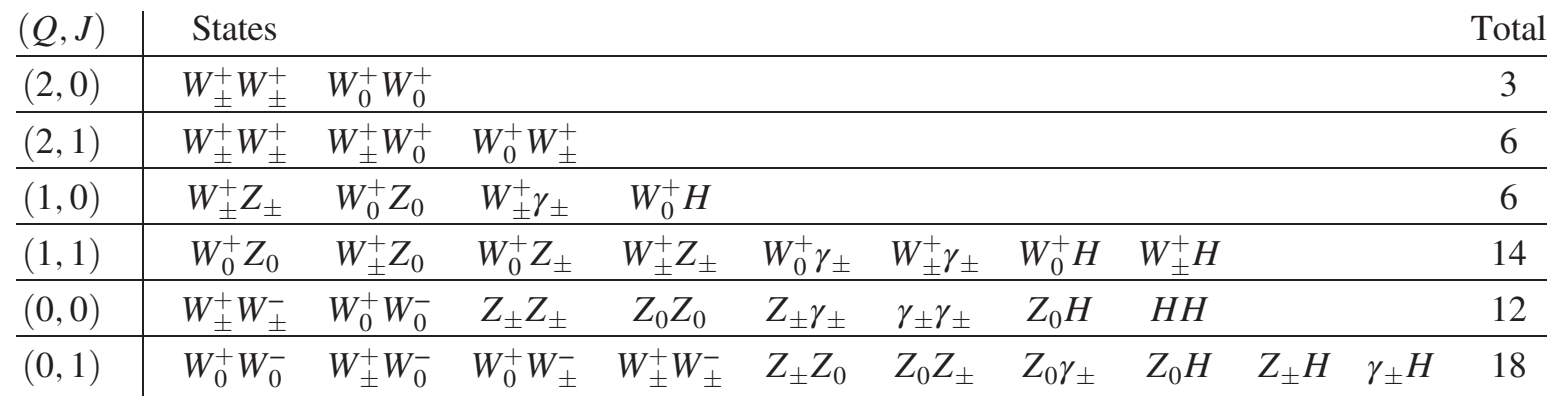


where upper indices indicate charge and lower indices helicity. We also display in Eq. (14) the dimensionality of the particle and helicity matrix for each independent $(Q, J)$ channel. Parity conservation at tree level leads to the reduction of the number of independent helicity amplitudes once we take into account the relation

$$
\begin{aligned}
& T^{J}\left(V_{1 \lambda_{1}} V_{2 \lambda_{2}} \rightarrow V_{3 \lambda_{3}} V_{4 \lambda_{4}}\right) \\
& \quad=(-1)^{\lambda_{1}-\lambda_{2}-\lambda_{3}+\lambda_{4}} T^{J}\left(V_{1-\lambda_{1}} V_{2-\lambda_{2}} \rightarrow V_{3-\lambda_{3}} V_{4-\lambda_{4}}\right) .
\end{aligned}
$$

Furthermore, time-reversal invariance also reduces the number of helicity amplitudes that need to be evaluated.

At this point, we would like to point out that there are further dimension-eight and $p^{4}$ nonlinear operators that can contribute to the processes listed in Eq. (14). For instance, the operators

$$
\begin{aligned}
& B^{\mu \nu} \Phi^{\dagger} \Phi\left(D_{\mu} \Phi\right)^{\dagger} D^{\mu} \Phi \quad \text { and } \\
& \operatorname{Tr}\left[D_{\mu} U U^{\dagger} D_{\nu} U U^{\dagger}\right] \partial^{\mu} \mathcal{F}_{8} \partial^{\nu} \mathcal{F}_{8}^{\prime}
\end{aligned}
$$

contribute to $V V \rightarrow H H$; for a complete list see Refs. [1,43,44]. Here, we focus on effective operators leading to genuine quartic gauge interactions, and we do not attempt to perform a full analysis for dimension-eight $\left(p^{4}\right)$ operators.

As an illustration, let us study the $Q=2$ and $J=0$ channel. The leading term in the center-of-mass energy $\sqrt{s}$ of the unitarity violating amplitudes is $\mathcal{O}\left(s^{2}\right)$ as expected from a naive dimensional analysis. ${ }^{1}$ Working in the basis $\left(W_{+}^{+} W_{+}^{+}, W_{0}^{+} W_{0}^{+}, W_{-}^{+} W_{-}^{+}\right)$, the $3 \times 3$ matrix in helicity particle space reads

$$
\frac{1}{96 \pi} \frac{s^{2}}{\Lambda^{4}}\left(\begin{array}{ccc}
6 f_{T_{1}}+3 f_{T_{2}} & 0 & 4 f_{T_{0}}+8 f_{T_{1}}+f_{T_{2}} \\
0 & 3 f_{S_{0}}+f_{S_{1}}+f_{S_{2}} & 0 \\
4 f_{T_{0}}+8 f_{T_{1}}+f_{T_{2}} & 0 & 6 f_{T_{1}}+3 f_{T_{2}}
\end{array}\right) .
$$

The strongest unitarity limits from this channel come from the eigenvalues of the above matrix:

$$
\begin{aligned}
\left|\frac{3 f_{S_{0}}+f_{S_{1}}+f_{S_{2}}}{\Lambda^{4}} s^{2}\right| & \leq 96 \pi, \quad\left|\frac{2 f_{T_{0}}+f_{T_{1}}-f_{T_{2}}}{\Lambda^{4}} s^{2}\right| \leq 48 \pi \\
\left|\frac{2 f_{T_{0}}+7 f_{T_{1}}+2 f_{T_{2}}}{\Lambda^{4}} s^{2}\right| & \leq 48 \pi .
\end{aligned}
$$

Clearly this allows us to constrain the coefficients only under the assumption of no cancellations between the different coefficients. So in order to obtain the most stringent bounds on the full set of coefficients, we diagonalize the six $T^{J}$ matrices and impose the constraint Eq. (13) on each of their eigenvalues.

\section{RESULTS}

We start our analysis by studying the operators that contain four covariant derivatives of the Higgs field, which are given in Eq. (2). The strongest unitarity limits for these operators originate from the $J=0$ partial wave. When we diagonalize the helicity-particle matrices for the three charges $(Q=0,1,2)$, we obtain three distinct nonvanishing eigenvalues given by

\footnotetext{
${ }^{1}$ Since genuine QGC do not have a TGC counterpart, gauge invariance does not lead to the cancellation of the $s^{2}$ terms [45], in contrast to what happens for dimension-six QGC [46].
}

$$
\begin{aligned}
& \frac{s^{2}}{96 \pi}\left(\frac{3 f_{S, 0}+f_{S, 1}+f_{S, 2}}{\Lambda^{4}}\right), \quad \frac{s^{2}}{96 \pi}\left(\frac{f_{S, 0}+f_{S, 1}+3 f_{S, 2}}{\Lambda^{4}}\right), \\
& \frac{s^{2}}{96 \pi}\left(\frac{3 f_{S, 0}+7 f_{S, 1}+5 f_{S, 2}}{\Lambda^{4}}\right),
\end{aligned}
$$

where we kept only the leading term in the center-of-mass energy.

These eigenvalues allow us to obtain limits on the three Wilson coefficients $f_{S, j} / \Lambda^{4}$. In order to explore the dependence of the bounds on the possible relations among operators imposed by specific forms of the ultraviolet physics, we consider two scenarios: In the first one, we assume that only one Wilson coefficient is nonvanishing. The second case assumes that all Wilson coefficients of the subset of operators considered are nonvanishing, and we look for the possible largest values of each coefficient in the unitarity region. Notice that in this second scenario, the limits for all couplings cannot be achieved simultaneously as they are simply extreme points in the three-dimension $\left(f_{S, 0} / \Lambda^{4}, f_{S, 1} / \Lambda^{4}, f_{S, 2} / \Lambda^{4}\right)$ region delimited by Eqs. (13) and (19). We present in Table I the bounds on $f_{S, j} / \Lambda^{4}$ for these two scenarios. Expectedly, the limits in the second case are weaker since the undisplayed Wilson coefficients can be adjusted to mitigate unitarity violation, but as explicitly shown, this is only an effect of $\mathcal{O}(1.5-4)$.

Next, we focus on the unitarity constraints on the seven operators $\mathcal{O}_{M j}$ from their leading contributions $\left[\mathcal{O}\left(s^{2}\right)\right]$ to the scattering amplitudes. For these operators, the analysis of the $J=0$ partial wave for the three charges yields two independent nonvanishing eigenvalues, 
TABLE I. Unitarity constraints on the Wilson coefficients of the $\mathcal{O}_{S, j}$ operators [Eq. (2)] when just one coefficient is nonvanishing (second and third columns), as well as when all coefficients are included (last two columns). For convenience, in the third and fifth columns, we give the numerical value of the bounds for maximal subprocess center-of-mass energy of 1.5 and $3 \mathrm{TeV}$.

\begin{tabular}{|c|c|c|c|c|}
\hline \multirow[b]{3}{*}{ Wilson coefficient } & \multicolumn{4}{|c|}{ Bound } \\
\hline & \multicolumn{2}{|c|}{1 operator } & \multicolumn{2}{|c|}{ All 3 operators } \\
\hline & & For $\sqrt{s}<1.5(3) \mathrm{TeV}$ & & For $\sqrt{s}<1.5(3) \mathrm{TeV}$ \\
\hline$\left|\frac{f_{S, 0}}{\Lambda^{4}}\right|$ & $32 \pi s^{-2}$ & $20(1.2) \mathrm{TeV}^{-4}$ & $48 \pi s^{-2}$ & $30(1.9) \mathrm{TeV}^{-4}$ \\
\hline$\left|\frac{f_{S, 1}}{\Delta^{4}}\right|$ & $\frac{96}{7} \pi s^{-2}$ & $8.5(0.53) \mathrm{TeV}^{-4}$ & $\frac{288 \pi}{5} s^{-2}$ & $35(2.2) \mathrm{TeV}^{-4}$ \\
\hline$\left|\frac{f_{S, 2}}{\Lambda^{4}}\right|$ & $\frac{96}{5} \pi s^{-2}$ & $8.5(0.53) \mathrm{TeV}^{-4}$ & $\frac{288 \pi}{5} s^{-2}$ & $35(2.2) \mathrm{TeV}^{-4}$ \\
\hline
\end{tabular}

$$
\begin{aligned}
& \frac{s^{2}}{64 \pi \Lambda^{4}}\left(2 f_{M, 4}+f_{M, 5}\right), \\
& \frac{s^{2}}{256 \pi \Lambda^{4}} \sqrt{32\left(-4 f_{M, 2}+f_{M, 3}\right)^{2}+6\left(8 f_{M, 0}-2 f_{M, 1}+f_{M, 7}\right)^{2}} .
\end{aligned}
$$

They allow for constraining the Wilson coefficients when considering only one operator at a time. Nevertheless, they are not enough to bound all coefficients in the most general scenario with several nonvanishing operators entering the amplitudes simultaneously. Consequently, to obtain the limits from the leading $\mathcal{O}\left(s^{2}\right)$ contribution, in this case one must also consider the bounds from the $J=1$ partial-wave unitarity. In so doing, we find seven additional independent nonzero eigenvalues in the $Q=2,1$ helicityparticle matrices,

$$
\begin{aligned}
& \frac{s^{2}}{1536 \pi \Lambda^{4}} C_{1,2,3}, \quad \frac{s^{2}}{6144 \pi \Lambda^{4}}\left(C_{4} \pm \sqrt{C_{5}^{2}+C_{6}^{2}}\right), \\
& \frac{s^{2}}{6144 \pi \Lambda^{4}}\left(C_{7} \pm \sqrt{C_{8}^{2}+C_{9}^{2}}\right)
\end{aligned}
$$

with

$$
\begin{aligned}
C_{1} & =12 f_{M, 0}+5 f_{M, 1}, \quad C_{2}=12 f_{M, 0}-11 f_{M, 1}+8 f_{M, 7}, \\
C_{3} & =4 f_{M, 7}, \\
C_{4,5} & = \pm\left(24 f_{M, 0}+10 f_{M, 1}-15 f_{M, 7}\right)+48 f_{M, 2}+20 f_{M, 3}, \\
C_{6} & =4 \sqrt{3}\left(6 f_{M, 4}-5 f_{M, 5}\right), \\
C_{7,8} & = \pm\left(-24 f_{M, 0}+22 f_{M, 1}-f_{M, 7}\right)-48 f_{M, 2}+44 f_{M, 3}, \\
C_{9} & =4 \sqrt{3}\left(6 f_{M, 4}+11 f_{M, 5}\right) .
\end{aligned}
$$

Altogether, the total number of unitarity constraints originating from the unitarity condition Eq. (13), with Eqs. (20) and (21), allow for independently bounding each of the $f_{M, j} / \Lambda^{4}$ Wilson coefficients even when all seven are considered simultaneously. In fact, due to the algebraic structure of the eigenvalues, it is technically possible to analytically solve the system of nine constraints in the

\begin{tabular}{|c|c|c|c|c|}
\hline \multirow[b]{3}{*}{ Wilson coefficient } & \multicolumn{4}{|c|}{ Bound } \\
\hline & \multicolumn{2}{|r|}{1 operator } & \multicolumn{2}{|c|}{ All 7 operators } \\
\hline & & For $\sqrt{s}<1.5(3) \mathrm{TeV}$ & & For $\sqrt{s}<1.5(3) \mathrm{TeV}$ \\
\hline$\left|\frac{f_{M, 0}}{\Lambda^{4}}\right|$ & $\frac{32}{\sqrt{6}} \pi s^{-2}$ & $8.1(0.5) \mathrm{TeV}^{-4}$ & $\left.\frac{2}{3}(72+5 \sqrt{6}) \pi\right) s^{-2}$ & $35(2.1) \mathrm{TeV}^{-4}$ \\
\hline$\left|\frac{f_{M, 1}}{\Lambda^{4}}\right|$ & $\frac{128}{\sqrt{6}} \pi s^{-2}$ & $32(2) \mathrm{TeV}^{-4}$ & $8\left(24+\frac{\sqrt{6}}{5}\right) \pi s^{-2}$ & $122(7.6) \mathrm{TeV}^{-4}$ \\
\hline$\left|\frac{f_{M, 2}}{\Lambda^{4}}\right|$ & $\frac{16}{\sqrt{2}} \pi s^{-2}$ & $7(0.44) \mathrm{TeV}^{-4}$ & $(24+5 \sqrt{2}) \pi s^{-2}$ & $20(1.3) \mathrm{TeV}^{-4}$ \\
\hline$\left|\frac{f_{M, 3}}{\Lambda^{4}}\right|$ & $\frac{64}{\sqrt{2}} \pi s^{-2}$ & 28(1.7) $\mathrm{TeV}^{-4}$ & $96 \pi s^{-2}$ & $60(3.7) \mathrm{TeV}^{-4}$ \\
\hline$\left|\frac{f_{M, 4}}{\Lambda^{4}}\right|$ & $32 \pi s^{-2}$ & $20(1.2) \mathrm{TeV}^{-4}$ & $4(5+8 \sqrt{3}) \pi s^{-2}$ & $58(3.6) \mathrm{TeV}^{-4}$ \\
\hline$\left|\frac{f_{M, 5}}{\Lambda^{4}}\right|$ & $64 \pi s^{-2}$ & $40(2.5) \mathrm{TeV}^{-4}$ & $64 \sqrt{3} \pi s^{-2}$ & $69(4.3) \mathrm{TeV}^{-4}$ \\
\hline$\left|\frac{f_{M, 7}}{\Lambda^{4}}\right|$ & $\frac{256}{\sqrt{6}} \pi s^{-2}$ & $65(4.0) \mathrm{TeV}^{-4}$ & $\frac{64}{5}(24+\sqrt{6}) \pi s^{-2}$ & $210(13) \mathrm{TeV}^{-4}$ \\
\hline
\end{tabular}
seven-dimensional parameter space.

We present in Table II the unitarity bounds on the Wilson coefficients of the operators $\mathcal{O}_{M, j}$ for the two scenarios described above. Even though the $J=1$ partial waves have to be invoked to obtained bounds in the full seven-dimensional parameter space and the limits of higher angular momentum amplitudes are weaker, it is interesting that the constraints on the Wilson coefficients do not

TABLE II. Same as Table I but for the operators $\mathcal{O}_{M, j}$ [Eq. (3)]. 
degrade substantially and become $\mathcal{O}(3-4)$ weaker than those obtained from the $J=0$ partial waves under the assumption of only one nonvanishing coefficient.

The third class of dimension-eight operators exhibits only field strength tensors, and it contains eight independent operators, given in Eq. (4). Considering only leading contributions to the scattering amplitudes that grow as $s^{2}$, the diagonalization of the $J=0$ helicity-particle matrices for the three $Q$ channels leads to eight distinct eigenvalues,

$$
\begin{aligned}
& \frac{s^{2}}{384 \pi \Lambda^{4}} D_{1,2,3,4}, \quad \frac{s^{2}}{384 \pi \Lambda^{4}}\left(D_{5} \pm \sqrt{D_{6}^{2}+D_{7}^{2}}\right), \\
& \frac{s^{2}}{384 \pi \Lambda^{4}}\left(D_{8} \pm \sqrt{D_{9}^{2}+D_{10}^{2}}\right)
\end{aligned}
$$

where

$$
\begin{aligned}
D_{1} & =8\left(2 f_{T, 0}+f_{T, 1}-f_{T, 2}\right), \\
D_{2} & =8\left(2 f_{T, 0}+7 f_{T, 1}+2 f_{T, 2}\right), \\
D_{3} & =8\left(2 f_{T, 5}+f_{T, 6}-f_{T, 7}\right), \\
D_{4} & =8\left(2 f_{T, 5}+7 f_{T, 6}+2 f_{T, 7}\right), \\
D_{5,6} & =4\left(2 f_{T, 0}+4 f_{T, 1}-f_{T, 2} \pm\left(8 f_{T, 8}-4 f_{T, 9}\right)\right), \\
D_{7} & =8 \sqrt{3} f_{T, 6}, \\
D_{8,9} & =80 f_{T, 0}+40 f_{T, 1}+26 f_{T, 2} \pm\left(128 f_{T, 8}+56 f_{T, 9}\right), \\
D_{10} & =4 \sqrt{3}\left(12 f_{T, 5}+2 f_{T, 6}+3 f_{T, 7}\right) .
\end{aligned}
$$

In this case the total number of unitarity constraints originating from the unitarity condition Eq. (13), together with the $J=0$ eigenvalues in Eq. (22), allow for independently bounding each of the $f_{T, j} / \Lambda^{4}$ Wilson coefficients even when the eight are considered simultaneously. And again, it is technically possible to analytically solve the system of eight constraints in the nine-dimensional parameter space. The bounds emanating from the $J=1$ partial wave are weaker than the ones from the $J=0$ one; therefore, we neglected them in this analysis.
We list in Table III the corresponding bounds for the $f_{T, j} / \Lambda^{4}$ coefficients assuming the two scenarios described above. Comparing the results in Tables I-III, we learn that the Wilson coefficients of the operators $\mathcal{O}_{T, j}$ are subject to stronger unitarity bounds than the other QGC classes. Moreover, for only one nonvanishing $f_{T, j} / \Lambda^{4}=1 \mathrm{TeV}^{-4}$, unitarity is not violated for subprocess center-of-mass energies smaller than 1.5-2.8 TeV, depending on the anomalous QGC.

We end by presenting the unitarity constraints on the $\mathcal{O}\left(p^{4}\right)$ QCG given in Eqs. (8) and (9) that originate in the nonlinear realization of the gauge symmetry. For this set of operators the most stringent limits stem from the $J=0$ partial wave. After diagonalizing the $Q=0,1,2$ channels, we obtain four non-vanishing eigenvalues:

$\frac{s^{2}}{24 v^{4} \pi} F_{1}, \quad \frac{s^{2}}{24 v^{4} \pi} F_{2}, \quad \frac{s^{2}}{24 v^{4} \pi}\left[F_{3} \pm \sqrt{8 F_{4}^{2}+F_{5}^{2}}\right]$,

where

$F_{1}=4\left(f_{P, 6}+2 f_{P, 11}\right)$,

$F_{2}=4\left(f_{P, 6}+2 f_{P, 11}+f_{P, 23}+2 f_{P, 24}\right)$,

$F_{3}=13 f_{P, 6}+11 f_{P, 11}+10 f_{P, 23}+10 f_{P, 24}+20 f_{P, 26}$,

$F_{4}=3 f_{P, 6}+f_{P, 11}+3 f_{P, 23}+f_{P, 24}$,

\begin{tabular}{|c|c|c|c|c|}
\hline \multirow[b]{3}{*}{ Wilson coefficient } & \multicolumn{4}{|c|}{ Bound } \\
\hline & \multicolumn{2}{|c|}{1 operator } & \multicolumn{2}{|c|}{ All 8 operators } \\
\hline & & For $\sqrt{s}<1.5(3) \mathrm{TeV}$ & & For $\sqrt{s}<1.5(3) \mathrm{TeV}$ \\
\hline$\left|\frac{f_{T, 0}}{\Lambda^{4}}\right|$ & $\frac{12}{5} \pi s^{-2}$ & $1.5(0.093) \mathrm{TeV}^{-4}$ & $\frac{136}{11} \pi s^{-2}$ & 7.7(0.48) $\mathrm{TeV}^{-4}$ \\
\hline$\left|\frac{f_{T, 1}}{\Lambda^{4}}\right|$ & $\frac{24}{5} \pi s^{-2}$ & $3.0(0.19) \mathrm{TeV}^{-4}$ & $\frac{352}{31} \pi s^{-2}$ & 7.0(0.44) $\mathrm{TeV}^{-4}$ \\
\hline$\left|\frac{f_{T, 2}}{\Lambda^{4}}\right|$ & $\frac{96}{13} \pi s^{-2}$ & 4.6(0.29) $\mathrm{TeV}^{-4}$ & $32 \pi s^{-2}$ & $20(1.2) \mathrm{TeV}^{-4}$ \\
\hline$\left|\frac{f_{T, 5}}{\Lambda^{4}}\right|$ & $\frac{8}{\sqrt{3}} \pi s^{-2}$ & 2.9(0.18) $\mathrm{TeV}^{-4}$ & $8\left(1+\frac{4 \sqrt{3}}{11}\right) \pi s^{-2}$ & 7.3(0.46) $\mathrm{TeV}^{-4}$ \\
\hline$\left|\frac{f_{T, 6}}{\Lambda^{4}}\right|$ & $\frac{48}{7} \pi s^{-2}$ & $4.1(0.27) \mathrm{TeV}^{-4}$ & $16\left(\frac{9+\sqrt{3}}{11}\right) \pi s^{-2}$ & $9.7(0.6) \mathrm{TeV}^{-4}$ \\
\hline$\left|\frac{f_{T, 7}}{\Lambda^{4}}\right|$ & $\frac{32}{\sqrt{3}} \pi s^{-2}$ & $11(0.72) \mathrm{TeV}^{-4}$ & $32\left(1+\frac{\sqrt{3}}{11}\right) \pi s^{-2}$ & 23(1.4) $\mathrm{TeV}^{-4}$ \\
\hline$\left|\frac{f_{T .8}}{\Lambda^{4}}\right|$ & $\frac{3}{2} \pi s^{-2}$ & $0.93(0.058) \mathrm{TeV}^{-4}$ & $\frac{18}{5} \pi s^{-2}$ & $2.2(0.14) \mathrm{TeV}^{-4}$ \\
\hline$\left|\frac{f_{T, 9}}{\Lambda^{4}}\right|$ & $\frac{24}{7} \pi s^{-2}$ & $2.1(0.13) \mathrm{TeV}^{-4}$ & $8 \pi s^{-2}$ & $5.0(0.31) \mathrm{TeV}^{-4}$ \\
\hline
\end{tabular}

$F_{5}=3 f_{P, 6}+f_{P, 11}-10 f_{P, 23}-10 f_{P, 24}-20 f_{P, 26}$.

Again, the structure of the four eigenvalues allows for independently constraining the five $f_{P, i}$ coefficients, even when considered to be all nonzero, simultaneously. Table IV contains the corresponding bounds on the coefficients. Notice that these results indicate that the present experimental analyses require the introduction of a unitarization procedure such as the one in Ref. [11].

TABLE III. Same as Table I but for the operators $\mathcal{O}_{T, j}$ [Eq. (4)]. 
TABLE IV. Same as Table I but for the operators in the nonlinear representation of the electroweak symmetry [Eqs. (8) and (9)].

\begin{tabular}{|c|c|c|c|c|}
\hline \multirow[b]{3}{*}{ Wilson coefficient } & \multicolumn{4}{|c|}{ Bound } \\
\hline & \multicolumn{2}{|c|}{1 operator } & \multicolumn{2}{|c|}{ All 5 operators } \\
\hline & & For $\sqrt{s}<1.5(3) \mathrm{TeV}$ & & For $\sqrt{s}<1.5(3) \mathrm{TeV}$ \\
\hline$\left|f_{P, 6}\right|$ & $\frac{12 \pi v^{4}}{11} S^{-2}$ & $2.6(0.15) \times 10^{-3}$ & $6 \pi v^{4} s^{-2}$ & $14(0.85) \times 10^{-3}$ \\
\hline$\left|f_{P, 11}\right|$ & $\frac{12 \pi v^{4}}{7} s^{-2}$ & $3.9(0.24) \times 10^{-3}$ & $6 \pi v^{4} s^{-2}$ & $14(0.85) \times 10^{-3}$ \\
\hline$\left|f_{P, 23}\right|$ & $\frac{12 \pi v^{4}}{5+\sqrt{43}} S^{-2}$ & $2.5(0.42) \times 10^{-3}$ & $12 \frac{(2+\sqrt{3}) \pi v^{4}}{5} s^{-2}$ & $22(1.4) \times 10^{-3}$ \\
\hline$\left|f_{P, 24}\right|$ & $\frac{12 \pi v^{4}}{5+3 \sqrt{3}} s^{-2}$ & $2.9(0.18) \times 10^{-3} u$ & $12 \frac{(2+\sqrt{3}) \pi v^{4}}{5} s^{-2}$ & $22(1.4) \times 10^{-3}$ \\
\hline$\left|f_{P, 26}\right|$ & $\frac{3 \pi v^{4}}{5} S^{-2}$ & $1.5(0.09) \times 10^{-3}$ & $\frac{12}{5} \pi v^{4} s^{-2}$ & $5.8(0.36) \times 10^{-3}$ \\
\hline
\end{tabular}

\section{DISCUSSION}

Exploration of the structure of the quartic couplings of electroweak gauge bosons is at the forefront of the tests of the SM, in general, and of its mechanism of symmetry breaking, in particular. Parametrizing deviations from the SM predictions in terms of effective operators is the standard methodology followed in such studies in the present experimental searches at the LHC [6-17]. Notwithstanding, the contribution of effective operators leads to unitarity violation at high energies, and therefore the methodology must be applied only in the energy regime in which this is not the case. For the specific case of genuine QGC operators, this has been partially addressed in the literature by studying the bounds imposed by partial-wave unitarity of gauge-boson scattering in specific channels and/or waves.

In this work we have presented a complete partial-wave analysis of two-to-two scattering of electroweak gauge bosons and Higgs bosons, all for the charged channels in Eq. (14). We have considered operator expansions with linear and nonlinear realizations of the electroweak symmetry. The leading anomalous contribution is proportional to $s^{2}$, and we studied the conditions to obtain the most stringent limits for all couplings.

Quantitatively, our results are summarize in Tables I-IV. In the minimal scenario with just one nonvanishing QCG Wilson coefficient, our analyses show that the strongest unitarity constraints can be obtained from the analyses of the $J=0$ partial wave for $Q=0,1,2$. However, in more realistic scenarios where more than one QGC operator contributes, the $J=0$ partial-wave analyses do not lead to the strongest unitarity bounds for all Wilson-coefficient combinations. In this case, we must also take into account the $J=1$ partial wave. Once all waves are considered, the bounds on each Wilson coefficient become a factor of a few weaker than in the minimal scenario.

\section{ACKNOWLEDGMENTS}

O. J.P.E. is supported in part by Conselho Nacional de Desenvolvimento Centífico e Tecnológico (CNPq) and by Fundação de Amparo à Pesquisa do Estado de São Paulo (FAPESP) Grant No. 2019/04837-9; E.S. A. thanks FAPESP for its support (Grant No. 2018/16921-1). M. C.G-G is supported by National Science Foundation Grant No. PHY-1620628, by the MINECO Grant No. FPA2016-76005-C2-1-P, by EU Grant No. FP10 ITN ELUSIVES (H2020-MSCA-ITN-2015-674896), and by AGAUR (Generalitat de Catalunya) Grant No. 2017SGR-929.

\section{APPENDIX: HELICITY AMPLITUDES}

Here, we present the list of unitarity violating amplitudes in Tables V-VIII for all the $2 \rightarrow 2$ scattering processes considered in the evaluation of the unitarity constraints. 
TABLE V. Unitarity violating (growing as $\left.s^{2}\right)$ terms of the scattering amplitudes $\mathcal{M}\left(V_{1 \lambda_{1}} V_{2 \lambda_{2}} \rightarrow V_{3 \lambda_{3}} V_{4 \lambda_{4}}\right)$ for longitudinal gauge bosons generated by the operators that contain four covariant derivatives of the Higgs field [Eq. (2)]. Here, $X \equiv \cos \theta$, and the overall factor extracted from all amplitudes is given at the top of the table.

\begin{tabular}{lc}
\hline \hline & $\times \frac{s^{2}}{\Lambda^{4}}$ \\
\hline$W^{+} W^{+} \rightarrow W^{+} W^{+}$ & $\left(4 f_{S, 0}+(1+X)\left(f_{S, 1}+f_{S, 2}\right)\right) / 8$ \\
$W Z \rightarrow W Z$ & $\left((5+3 X) f_{S, 0}-2(-1+X) f_{S, 1}+(5+3 X) f_{S, 2}\right) / 16$ \\
$W Z \rightarrow W H$ & $-\left((-1+X)(3+X)\left(f_{S, 0}-f_{S, 2}\right)\right) / 16$ \\
$W H \rightarrow W H$ & $\left((5+3 X) f_{S, 0}-2(-1+X) f_{S, 1}+(5+3 X) f_{S, 2}\right) / 16$ \\
$W^{+} W^{-} \rightarrow W^{+} W^{-}$ & $\left((2+6 X) f_{S, 0}-(-5+X)\left(f_{S, 1}+f_{S, 2}\right)\right) / 8$ \\
$W^{+} W^{-} \rightarrow Z Z$ & $\left(f_{S, 0}+X f_{S, 0}+4 f_{S, 1}+f_{S, 2}+X f_{S, 2}\right) /(8 \sqrt{2})$ \\
$W^{+} W^{-} \rightarrow Z H$ & $-\left(X\left(f_{S, 0}-f_{S, 2}\right)\right) / 4$ \\
$W^{+} W^{-} \rightarrow H H$ & $-\left(f_{S, 0}+4 f_{S, 1}+f_{S, 2}+X^{2}\left(f_{S, 0}+f_{S, 2}\right)\right) /(8 \sqrt{2})$ \\
$Z Z \rightarrow Z Z$ & $\left((3+X)\left(f_{S, 0}+f_{S, 1}+f_{S, 2}\right)\right) / 8$ \\
$Z Z \rightarrow H H$ & $\left(f_{S, 0}-X^{2} f_{S, 0}-2\left(f_{S, 1}+f_{S, 2}\right)\right) / 8$ \\
$Z H \rightarrow Z H$ & $\left(4(1+X) f_{S, 0}-(-1+X)\left(f_{S, 1}+f_{S, 2}\right)\right) / 8$ \\
$H H \rightarrow H H$ & $(3+X)\left(f_{S, 0}+f_{S, 1}+f_{S, 2}\right) / 8$ \\
\hline \hline
\end{tabular}

TABLE VI. Unitarity violating (growing as $\left.s^{2}\right)$ terms of the scattering amplitudes $\mathcal{M}\left(V_{1 \lambda_{1}} V_{2 \lambda_{2}} \rightarrow V_{3 \lambda_{3}} V_{4 \lambda_{4}}\right)$ for the gauge boson helicities given in the second column generated by the operators that contain two covariant derivatives of the Higgs field [Eq. (3)]. Here, $X \equiv \cos \theta, X_{P} \equiv 1+\cos \theta, X_{M} \equiv 1-\cos \theta, c_{W}=\cos \theta_{W}, s_{W}=\sin \theta_{W}, c_{2 W}=\cos 2 \theta_{W}, c_{4 W}=\cos 4 \theta_{W}$, and $s_{2 W}=\sin 2 \theta_{W}$, and the overall factor extracted from all amplitudes is given at the top of the table.

\begin{tabular}{|c|c|c|}
\hline & & $\times \frac{s^{2}}{\Lambda^{4}}$ \\
\hline$W^{+} W^{+} \rightarrow W^{+} W^{+}$ & $\begin{array}{l}0+0- \\
0+0+ \\
+00- \\
+00+\end{array}$ & $\begin{array}{c}-X_{M}\left(-4 X_{M} f_{M, 0}+X_{M} f_{M, 1}-2 f_{M, 7}\right) / 64 \\
X_{P}\left(2 f_{M, 1}-f_{M, 7}\right) / 32 \\
-X_{P}\left(4 X_{P} f_{M, 0}-X_{P} f_{M, 1}+2 f_{M, 7}\right) / 64 \\
-X_{M}\left(2 f_{M, 1}-f_{M, 7}\right) / 32\end{array}$ \\
\hline$W^{+} Z \rightarrow W^{+} Z$ & $\begin{array}{l}00+- \\
00++ \\
0+0- \\
0+0+ \\
+00- \\
+00+\end{array}$ & $\begin{array}{c}X_{M} X_{P} f_{M, 5} s_{W} / 16 \\
X f_{M, 7} c_{W}+2\left(2 f_{M, 4}+f_{M, 5}\right) s_{W} / 16 \\
X_{M}^{2}\left(\left(16 f_{M, 2}-4 f_{M, 3}\right) s_{W}^{2}+\left(8 f_{M, 0}-2 f_{M, 1}+f_{M, 7}\right) c_{W}^{2}-2\left(2 f_{M, 4}+f_{M, 5}\right) s_{2 W}\right) / 64 \\
X_{P}\left(4 f_{M, 3} s_{W}^{2}+\left(2 f_{M, 1}-f_{M, 7}\right) c_{W}^{2}+2 f_{M, 5} s_{2 W}\right) / 16 \\
X_{P}\left((-3+X) f_{M, 7} c_{W}-2 X_{P}\left(2 f_{M, 4}+f_{M, 5}\right) s_{W}\right) / 64 \\
X_{M} f_{M, 5} s_{W} / 8\end{array}$ \\
\hline$W^{+} Z \rightarrow W^{+} \gamma$ & $\begin{array}{l}00+- \\
00++ \\
0+0- \\
0+0+ \\
+00- \\
+00+\end{array}$ & $\begin{array}{c}\left.-X_{M} X_{P} f_{M, 5} c_{W}\right) / 16 \\
\left(-2\left(2 f_{M, 4}+f_{M, 5}\right) c_{W}+X f_{M, 7} s_{W}\right) / 16 \\
X_{M}^{2}\left(4\left(2 f_{M, 4}+f_{M, 5}\right) c_{2 W}+\left(8 f_{M, 0}-2 f_{M, 1}-16 f_{M, 2}+4 f_{M, 3}+f_{M, 7}\right) s_{2 W}\right) / 128 \\
-X_{P}\left(4 f_{M, 5} c_{2 W}+\left(-2 f_{M, 1}+4 f_{M, 3}+f_{M, 7}\right) s_{2 W}\right) / 32 \\
X_{P}\left(2 X_{P}\left(2 f_{M, 4}+f_{M, 5}\right) c_{W}+(-3+X) f_{M, 7} s_{W}\right) / 64 \\
-X_{M} f_{M, 5} c_{W} / 8\end{array}$ \\
\hline$W^{+} Z \rightarrow W^{+} H$ & $\begin{array}{l}0+-0 \\
0++0 \\
+-00 \\
+0-0 \\
++00\end{array}$ & $\begin{array}{c}X_{P}\left((-3+X) f_{M, 7} c_{W}-2 X_{P}\left(2 f_{M, 4}+f_{M, 5}\right) s_{W}\right) / 64 \\
X_{M} f_{M, 5} s_{W} / 8 \\
-X_{M} X_{P} f_{M, 5} s_{W} / 16 \\
-X_{M}(3+X) f_{M, 7} / 64 \\
\left(-\left(X f_{M, 7} c_{W}\right)-2\left(2 f_{M, 4}+f_{M, 5}\right) s_{W}\right) / 16\end{array}$ \\
\hline$W^{+} \gamma \rightarrow W^{+} \gamma$ & $\begin{array}{l}0+0- \\
0+0+\end{array}$ & $\begin{array}{c}-X_{M}^{2}\left(\left(-8 f_{M, 0}+2 f_{M, 1}-f_{M, 7}\right) s_{W}^{2}+\left(-16 f_{M, 2}+4 f_{M, 3}\right) c_{W}^{2}-2\left(2 f_{M, 4}+f_{M, 5}\right) s_{2 W}\right) / 64 \\
X_{P}\left(\left(2 f_{M, 1}-f_{M, 7}\right) s_{W}^{2}+4 f_{M, 3} c_{W}^{2}-2 f_{M, 5} s_{2 W}\right) / 16\end{array}$ \\
\hline$W^{+} \gamma \rightarrow W^{+} H$ & $\begin{array}{l}0+-0 \\
0++0 \\
+-00 \\
++00\end{array}$ & $\begin{array}{c}X_{P}\left(2 X_{P}\left(2 f_{M, 4}+f_{M, 5}\right) c_{W}+(-3+X) f_{M, 7} s_{W}\right) / 64 \\
-X_{M} f_{M, 5} c_{W} / 8 \\
X_{M} X_{P} f_{M, 5} c_{W} / 16 \\
\left(2\left(2 f_{M, 4}+f_{M, 5}\right) c_{W}-X f_{M, 7} s_{W}\right) / 16\end{array}$ \\
\hline
\end{tabular}




\begin{tabular}{|c|c|c|}
\hline & & $\times \frac{s^{2}}{\Lambda^{4}}$ \\
\hline$W^{+} H \rightarrow W^{+} H$ & $\begin{array}{l}+0-0 \\
+0+0\end{array}$ & $\begin{array}{c}X_{M}^{2}\left(8 f_{M, 0}-2 f_{M, 1}+f_{M, 7}\right) / 64 \\
X_{P}\left(2 f_{M, 1}-f_{M, 7}\right) / 16\end{array}$ \\
\hline$W^{+} W^{-} \rightarrow W^{+} W^{-}$ & $\begin{array}{l}00+- \\
00++ \\
0+0- \\
0+0+\end{array}$ & $\begin{array}{c}-X_{M} X_{P}\left(2 f_{M, 1}-f_{M, 7}\right) / 32 \\
\left(8 f_{M, 0}-2 f_{M, 1}+f_{M, 7}+X f_{M, 7}\right) / 16 \\
-X_{M}\left(-4 X_{M} f_{M, 0}+f_{M, 1}-X f_{M, 1}+f_{M, 7}+X f_{M, 7}\right) / 32 \\
X_{P}\left(2 f_{M, 1}-f_{M, 7}\right) / 16\end{array}$ \\
\hline$W^{+} W^{-} \rightarrow Z Z$ & $\begin{array}{l}00+- \\
00++ \\
0+0- \\
0+0+ \\
+00- \\
+00+\end{array}$ & $\begin{array}{c}X_{M} X_{P}\left(\left(-2 f_{M, 1}+f_{M, 7}\right) c_{W}^{2}+4 f_{M, 3} s_{W}^{2}+2 f_{M, 5} s_{2 W}\right) /(32 \sqrt{2}) \\
\left(\left(16 f_{M, 2}-4 f_{M, 3}\right) s_{W}^{2}+\left(8 f_{M, 0}-2 f_{M, 1}+f_{M, 7}\right) c_{W}^{2}-2\left(2 f_{M, 4}+f_{M, 5}\right) s_{2 W}\right) /(16 \sqrt{2}) \\
-X_{M}\left((3+X) f_{M, 7} c_{W}-2 X_{M}\left(2 f_{M, 4}+f_{M, 5}\right) s_{W}\right) /(64 \sqrt{2}) \\
-X_{P} f_{M, 5} s_{W} /(8 \sqrt{2}) \\
-X_{P}\left((-3+X) f_{M, 7} c_{W}+2 X_{P}\left(2 f_{M, 4}+f_{M, 5}\right) s_{W}\right) /(64 \sqrt{2}) \\
X_{M} f_{M, 5} s_{W} /(8 \sqrt{2})\end{array}$ \\
\hline$W^{+} W^{-} \rightarrow Z \gamma$ & $\begin{array}{l}00+- \\
00++ \\
0+0- \\
0+0+ \\
+00- \\
+00+\end{array}$ & $\begin{array}{c}X_{M} X_{P}\left(4 f_{M, 5} c_{2 W}+\left(-2 f_{M, 1}+4 f_{M, 3}+f_{M, 7}\right) s_{2 W}\right) / 64 \\
\left(2\left(2 f_{M, 4}+f_{M, 5}\right) c_{2 W}+\left(8 f_{M, 0}-2 f_{M, 1}-16 f_{M, 2}+4 f_{M, 3}+f_{M, 7}\right) c_{W} s_{W}\right) / 16 \\
X_{M}\left(-2 X_{M}\left(2 f_{M, 4}+f_{M, 5}\right) c_{W}-(3+X) f_{M, 7} s_{W}\right) / 64 \\
X_{P} f_{M, 5} c_{W} / 8 \\
X_{P}\left(2 X_{P}\left(2 f_{M, 4}+f_{M, 5} c_{W}-(-3+X) f_{M, 7} s_{W}\right) / 64\right. \\
-X_{M} f_{M, 5} c_{W} / 8\end{array}$ \\
\hline$W^{+} W^{-} \rightarrow Z H$ & $\begin{array}{l}0+-0 \\
0+10 \\
+0-0 \\
+0+0 \\
++00\end{array}$ & $\begin{array}{c}-X_{P}\left((-3+X) f_{M, 7} c_{W}+2 X_{P}\left(2 f_{M, 4}+f_{M, 5}\right) s_{W}\right) / 64 \\
X_{M} f_{M, 5} s_{W} / 8 \\
X_{M}\left((3+X) f_{M, 7} c_{W}-2 X_{M}\left(2 f_{M, 4}+f_{M, 5}\right) s_{W}\right) / 64 \\
X_{P} f_{M, 5} s_{W} / 8 \\
X f_{M, 7} / 16\end{array}$ \\
\hline$W^{+} W^{-} \rightarrow \gamma \gamma$ & $\begin{array}{l}00+- \\
00++\end{array}$ & $\begin{array}{c}-X_{M} X_{P}\left(\left(2 f_{M, 1}-f_{M, 7}\right) s_{W}^{2}+4 f_{M, 3} c_{W}^{2}-2 f_{M, 5} s_{2 W}\right) /(32 \sqrt{2}) \\
\left(\left(8 f_{M, 0}-2 f_{M, 1}+f_{M, 7}\right) s_{W}^{2}-\left(-16 f_{M, 2}+4 f_{M, 3}\right) c_{W}^{2}+2\left(2 f_{M, 4}+f_{M, 5}\right) s_{2 W}\right) /(16 \sqrt{2})\end{array}$ \\
\hline$W^{+} W^{-} \rightarrow \gamma H$ & $\begin{array}{l}0+-0 \\
0+10 \\
+0-0 \\
+0+0\end{array}$ & $\begin{array}{c}X_{P}\left(2 X_{P}\left(2 f_{M, 4}+f_{M, 5}\right) c_{W}-(-3+X) f_{M, 7} s_{W}\right) / 64 \\
-X_{M} f_{M, 5} c_{W} / 8 \\
-X_{M}\left(-2 X_{M}\left(2 f_{M, 4}+f_{M, 5}\right) c_{W}-(3+X) f_{M, 7} s_{W}\right) / 64 \\
-X_{P} f_{M, 5} c_{W} / 8\end{array}$ \\
\hline$W^{+} W^{-} \rightarrow H H$ & $\begin{array}{l}+-00 \\
++00\end{array}$ & $\begin{aligned} & X_{M} X_{P}\left(2 f_{M, 1}-f_{M, 7}\right) /(32 \sqrt{2}) \\
- & \left(8 f_{M, 0}-2 f_{M, 1}+f_{M, 7}\right) /(16 \sqrt{2})\end{aligned}$ \\
\hline$Z Z \rightarrow Z Z$ & $\begin{array}{l}00+- \\
00++ \\
0+0- \\
0+0+ \\
+00- \\
+00+\end{array}$ & $\begin{array}{c}X_{M} X_{P}\left(-4 f_{M, 3} s_{W}^{2}+\left(-2 f_{M, 1}+f_{M, 7}\right) c_{W}^{2}+2 f_{M, 5} s_{2 W}\right) / 64 \\
\left(\left(16 f_{M, 2}-4 f_{M, 3}\right) s_{W}^{2}+\left(8 f_{M, 0}-2 f_{M, 1}+f_{M, 7}\right) c_{W}^{2}+2\left(2 f_{M, 4}+f_{M, 5}\right) s_{2 W}\right) / 32 \\
X_{M}^{2}\left(\left(16 f_{M, 2}-4 f_{M, 3}\right) s_{W}^{2}+\left(8 f_{M, 0}-2 f_{M, 1}+f_{M, 7}\right) c_{W}^{2}+2\left(2 f_{M, 4}+f_{M, 5}\right) s_{2 W}\right) / 128 \\
-X_{P}\left(-4 f_{M, 3} s_{W}^{2}+\left(-2 f_{M, 1}+f_{M, 7}\right) c_{W}^{2}+2 f_{M, 5} s_{2 W}\right) / 32 \\
-X_{P}^{2}\left(\left(16 f_{M, 2}-4 f_{M, 3}\right) s_{W}^{2}+\left(8 f_{M, 0}-2 f_{M, 1}+f_{M, 7}\right) c_{W}^{2}+2\left(2 f_{M, 4}+f_{M, 5}\right) s_{2 W}\right) / 128 \\
X_{M}\left(-4 f_{M, 3} s_{W}^{2}+\left(-2 f_{M, 1}+f_{M, 7}\right) c_{W}^{2}+2 f_{M, 5} s_{2 W}\right) / 32\end{array}$ \\
\hline$Z Z \rightarrow Z \gamma$ & $\begin{array}{l}00+- \\
00++ \\
0+0- \\
0+0+ \\
+00- \\
+00+\end{array}$ & $\begin{array}{c}-X_{M} X_{P}\left(4 f_{M, 5} c_{2 W}+\left(2 f_{M, 1}-4 f_{M, 3}-f_{M, 7}\right) s_{2 W}\right) /(64 \sqrt{2}) \\
\left(-2\left(2 f_{M, 4}+f_{M, 5}\right) c_{2 W}+\left(8 f_{M, 0}-2 f_{M, 1}-16 f_{M, 2}+4 f_{M, 3}+f_{M, 7}\right) c_{W} s_{W}\right) /(16 \sqrt{2}) \\
-X_{M}^{2}\left(4\left(2 f_{M, 4}+f_{M, 5}\right) c_{2 W}-\left(8 f_{M, 0}-2 f_{M, 1}-16 f_{M, 2}+4 f_{M, 3}+f_{M, 7}\right) s_{2 W}\right) /(128 \sqrt{2}) \\
X_{P}\left(4 f_{M, 5} c_{2 W}+\left(2 f_{M, 1}-4 f_{M, 3}-f_{M, 7}\right) s_{2 W}\right) /(32 \sqrt{2}) \\
X_{P}^{2}\left(4\left(2 f_{M, 4}+f_{M, 5}\right) c_{2 W}-\left(8 f_{M, 0}-2 f_{M, 1}-16 f_{M, 2}+4 f_{M, 3}+f_{M, 7}\right) s_{2 W}\right) /(128 \sqrt{2}) \\
-X_{M}\left(4 f_{M, 5} c_{2 W}+\left(2 f_{M, 1}-4 f_{M, 3}-f_{M, 7}\right) s_{2 W}\right) /(32 \sqrt{2})\end{array}$ \\
\hline$Z Z \rightarrow \gamma \gamma$ & $\begin{array}{l}00+- \\
00++\end{array}$ & $\begin{array}{c}-X_{M} X_{P}\left(\left(2 f_{M, 1}-f_{M, 7}\right) s_{W}^{2}+4 f_{M, 3} c_{W}^{2}+2 f_{M, 5} s_{2 W}\right) / 64 \\
\left(\left(8 f_{M, 0}-2 f_{M, 1}+f_{M, 7}\right) s_{W}^{2}-\left(-16 f_{M, 2}+4 f_{M, 3}\right) c_{W}^{2}-2\left(2 f_{M, 4}+f_{M, 5}\right) s_{2 W}\right) / 32\end{array}$ \\
\hline$Z Z \rightarrow H H$ & $\begin{array}{l}+-00 \\
++00\end{array}$ & $\begin{array}{c}-X_{M} X_{P}\left(-4 f_{M, 3} s_{W}^{2}+\left(-2 f_{M, 1}+f_{M, 7}\right) c_{W}^{2}+2 f_{M, 5} s_{2 W}\right) / 64 \\
\left(4\left(-4 f_{M, 2}+f_{M, 3}\right) s_{W}^{2}-\left(8 f_{M, 0}-2 f_{M, 1}+f_{M, 7}\right) c_{W}^{2}-2\left(2 f_{M, 4}+f_{M, 5}\right) s_{2 W}\right) / 32\end{array}$ \\
\hline
\end{tabular}


TABLE VI. (Continued)

\begin{tabular}{lcc}
\hline \hline & & $\times \frac{s^{2}}{\Lambda^{4}}$ \\
\hline$Z \gamma \rightarrow Z \gamma$ & $0+0-$ & $-X_{M}^{2}\left(\left(-8 f_{M, 0}+2 f_{M, 1}-f_{M, 7}\right) s_{W}^{2}+\left(-16 f_{M, 2}+4 f_{M, 3}\right) c_{W}^{2}+2\left(2 f_{M, 4}+f_{M, 5}\right) s_{2 W}\right) / 64$ \\
& $0+0+$ & $X_{P}\left(\left(2 f_{M, 1}-f_{M, 7}\right) s_{W}^{2}+4 f_{M, 3} c_{W}^{2}+2 f_{M, 5} s_{2 W}\right) / 16$ \\
$Z \gamma \rightarrow H H$ & +-00 & $X_{M} X_{P}\left(4 f_{M, 5} c_{2 W}+\left(2 f_{M, 1}-4 f_{M, 3}-f_{M, 7}\right) s_{2 W}\right) /(64 \sqrt{2})$ \\
& ++00 & $\left(2\left(2 f_{M, 4}+f_{M, 5}\right) c_{2 W}-\left(8 f_{M, 0}-2 f_{M, 1}-16 f_{M, 2}+4 f_{M, 3}+f_{M, 7}\right) c_{W} s_{W}\right) /(16 \sqrt{2})$ \\
$Z H \rightarrow Z H$ & $+0-0$ & $\left.X_{M}^{2}\left(\left(16 f_{M, 2}-4 f_{M, 3}\right) s_{W}^{2}+\left(8 f_{M, 0}-2 f_{M, 1}+f_{M, 7}\right) c_{W}^{2}+2\left(2 f_{M, 4}+f_{M, 5}\right) s_{2 W}\right)\right) / 64$ \\
& $+0+0$ & $-X_{P}\left(-4 f_{M, 3} s_{W}^{2}+\left(-2 f_{M, 1}+f_{M, 7}\right) c_{W}^{2}+2 f_{M, 5} s_{2 W}\right) / 16$ \\
$Z H \rightarrow \gamma H$ & $+0-0$ & $-X_{M}^{2}\left(4\left(2 f_{M, 4}+f_{M, 5}\right) c_{2 W}-\left(8 f_{M, 0}-2 f_{M, 1}-16 f_{M, 2}+4 f_{M, 3}+f_{M, 7}\right) s_{2 W}\right) / 128$ \\
$\gamma \gamma \rightarrow H H$ & $+0+0$ & $X_{P}\left(4 f_{M, 5} c_{2 W}+\left(2 f_{M, 1}-4 f_{M, 3}-f_{M, 7}\right) s_{2 W}\right) / 32$ \\
& +-00 & $-X_{M} X_{P}\left(\left(-2 f_{M, 1}+f_{M, 7}\right) s_{W}^{2}-4 f_{M, 3} c_{W}^{2}-2 f_{M, 5} s_{2 W}\right) / 64$ \\
$\gamma H \rightarrow \gamma H$ & ++00 & $\left(4\left(-4 f_{M, 2}+f_{M, 3}\right) c_{W}^{2}+\left(2\left(2 f_{M, 4}+f_{M, 5}\right) s_{2 W}-\left(8 f_{M, 0}-2 f_{M, 1}+f_{M, 7}\right) s_{W}^{2}\right) / 32\right.$ \\
$\gamma H \rightarrow \gamma H$ & $+0-0$ & $-X_{M}^{2}\left(\left(-8 f_{M, 0}+2 f_{M, 1}-f_{M, 7}\right) s_{W}^{2}+\left(-16 f_{M, 2}+4 f_{M, 3}\right) c_{W}^{2}+2\left(2 f_{M, 4}+f_{M, 5}\right) s_{2 W}\right) / 64$ \\
\hline \hline
\end{tabular}

TABLE VII. Same as Table VI but for the operators containing only field strength tensors [Eq. (4)].

\begin{tabular}{|c|c|c|}
\hline & & $\times \frac{s^{2}}{\Lambda^{4}}$ \\
\hline$W^{+} W^{+} \rightarrow W^{+} W^{+}$ & $\begin{array}{l}+--+ \\
+-+- \\
++-- \\
++++\end{array}$ & $\begin{array}{c}X_{M}^{2}\left(2 f_{T, 0}+f_{T, 1}+f_{T, 2}\right) / 8 \\
X_{P}^{2}\left(2 f_{T, 0}+f_{T, 1}+f_{T, 2}\right) / 8 \\
\left(4 X_{P} f_{T, 0}+2(5+X) f_{T, 1}+X_{P} f_{T, 2}\right) / 8 \\
f_{T, 1}+f_{T, 2} / 2\end{array}$ \\
\hline$W^{+} Z \rightarrow W^{+} Z$ & $\begin{array}{l}+--+ \\
+-+- \\
++-- \\
++++\end{array}$ & $\begin{array}{c}\left.X_{M}^{2}\left(\left(4 f_{T, 5}+f_{T, 7}\right) s_{W}^{2}+\left(4 f_{T, 0}+f_{T, 2}\right) c_{W}^{2}\right)\right) / 8 \\
X_{P}^{2}\left(\left(2 f_{T, 6}+f_{T, 7}\right) s_{W}^{2}+\left(2 f_{T, 1}+f_{T, 2}\right) c_{W}^{2}\right) / 8 \\
\left(\left(4 X_{M} f_{T, 5}+10 f_{T, 6}+6 X f_{T, 6}+X_{M} f_{T, 7}\right) s_{W}^{2}+\left(4 X_{M} f_{T, 0}+10 f_{T, 1}+6 X f_{T, 1}+X_{M} f_{T, 2}\right) c_{W}^{2}\right) / 8 \\
\left(\left(2 f_{T, 6}+f_{T, 7}\right) s_{W}^{2}+\left(2 f_{T, 1}+f_{T, 2}\right) c_{W}^{2}\right) / 2\end{array}$ \\
\hline$W^{+} Z \rightarrow W^{+} \gamma$ & $\begin{array}{l}+--+ \\
+-+- \\
++-- \\
++++\end{array}$ & $\begin{array}{c}X_{M}^{2}\left(4 f_{T, 0}+f_{T, 2}-4 f_{T, 5}-f_{T, 7}\right) s_{2 W} / 16 \\
X_{P}^{2}\left(2 f_{T, 1}+f_{T, 2}-2 f_{T, 6}-f_{T, 7}\right) s_{2 W} / 16 \\
-\left(-4 X_{M} f_{T, 0}-2(5+3 X) f_{T, 1}-X_{M} f_{T, 2}+4 X_{M} f_{T, 5}+10 f_{T, 6}+6 X f_{T, 6}+X_{M} f_{T, 7}\right) s_{2 W} / 16 \\
\left(2 f_{T, 1}+f_{T, 2}-2 f_{T, 6}-f_{T, 7}\right) s_{2 W} / 4\end{array}$ \\
\hline$W^{+} \gamma \rightarrow W^{+} \gamma$ & $\begin{array}{l}+--+ \\
+-+- \\
++-- \\
++++\end{array}$ & $\begin{array}{c}X_{M}^{2}\left(\left(4 f_{T, 0}+f_{T, 2}\right) s_{W}^{2}+\left(4 f_{T, 5}+f_{T, 7}\right) c_{W}^{2}\right) / 8 \\
X_{P}^{2}\left(\left(2 f_{T, 1}+f_{T, 2}\right) s_{W}^{2}+\left(2 f_{T, 6}+f_{T, 7}\right) c_{W}^{2}\right) / 8 \\
\left(\left(4 X_{M} f_{T, 0}+10 f_{T, 1}+6 X f_{T, 1}+X_{M} f_{T, 2}\right) s_{W}^{2}+\left(4 X_{M} f_{T, 5}+10 f_{T, 6}+6 X f_{T, 6}+X_{M} f_{T, 7}\right) c_{W}^{2}\right) / 8 \\
\left(\left(2 f_{T, 1}+f_{T, 2}\right) s_{W}^{2}+\left(2 f_{T, 6}+f_{T, 7}\right) c_{W}^{2}\right) / 2\end{array}$ \\
\hline$W^{+} W^{-} \rightarrow W^{+} W^{-}$ & $\begin{array}{l}+--+ \\
+-+- \\
++-- \\
++++\end{array}$ & $\begin{array}{c}X_{M}^{2}\left(2 f_{T, 0}+f_{T, 1}+f_{T, 2}\right) / 4 \\
X_{P}^{2}\left(2 f_{T, 1}+f_{T, 2}\right) / 4 \\
\left(-4(-5+X) f_{T, 0}+2(7+5 X) f_{T, 1}-(-5+X) f_{T, 2}\right) / 8 \\
2 f_{T, 0}+f_{T, 1}+f_{T, 2}\end{array}$ \\
\hline$W^{+} W^{-} \rightarrow Z Z$ & $\begin{array}{l}+--+ \\
+-+- \\
++-- \\
++++\end{array}$ & $\begin{array}{c}X_{M}^{2}\left(\left(2 f_{T, 6}+f_{T, 7}\right) s_{W}^{2}+\left(2 f_{T, 1}+f_{T, 2}\right) c_{W}^{2}\right) /(8 \sqrt{2}) \\
X_{P}^{2}\left(\left(2 f_{T, 6}+f_{T, 7}\right) s_{W}^{2}+\left(2 f_{T, 1}+f_{T, 2}\right) c_{W}^{2}\right) /(8 \sqrt{2}) \\
\left(\left(4 f_{T, 5}+X_{P} f_{T, 6}+f_{T, 7}\right) s_{W}^{2}+\left(4 f_{T, 0}+X_{P} f_{T, 1}+f_{T, 2}\right) c_{W}^{2}\right) /(2 \sqrt{2}) \\
\left(\left(4 f_{T, 5}+f_{T, 7}\right) s_{W}^{2}+\left(4 f_{T, 0}+f_{T, 2}\right) c_{W}^{2}\right) /(2 \sqrt{2})\end{array}$ \\
\hline$W^{+} W^{-} \rightarrow Z \gamma$ & $\begin{array}{l}+--+ \\
+-+- \\
++-- \\
++++\end{array}$ & $\begin{array}{c}X_{M}^{2}\left(2 f_{T, 1}+f_{T, 2}-2 f_{T, 6}-f_{T, 7}\right) s_{2 W} / 16 \\
X_{P}^{2}\left(2 f_{T, 1}+f_{T, 2}-2 f_{T, 6}-f_{T, 7}\right) s_{2 W} / 16 \\
\left(4 f_{T, 0}+X_{P} f_{T, 1}+f_{T, 2}-4 f_{T, 5}-X_{M} f_{T, 6}-f_{T, 7}\right) s_{2 W} / 4 \\
\left(4 f_{T, 0}+f_{T, 2}-4 f_{T, 5}-f_{T, 7}\right) s_{2 W} / 4\end{array}$ \\
\hline
\end{tabular}


TABLE VII. (Continued)

\begin{tabular}{|c|c|c|}
\hline & & $\times \frac{s^{2}}{\Lambda^{4}}$ \\
\hline$W^{+} W^{-} \rightarrow \gamma \gamma$ & $\begin{array}{l}+--+ \\
+-+- \\
++-- \\
++++\end{array}$ & $\begin{array}{c}X_{M}^{2}\left(\left(2 f_{T, 1}+f_{T, 2}\right) s_{W}^{2}+\left(2 f_{T, 6}+f_{T, 7}\right) c_{W}^{2}\right) /(8 \sqrt{2}) \\
\left.X_{P}^{2}\left(\left(2 f_{T, 1}+f_{T, 2}\right) s_{W}^{2}+\left(2 f_{T, 6}+f_{T, 7}\right) c_{W}^{2}\right)\right) /(8 \sqrt{2}) \\
\left(\left(4 f_{T, 0}+X_{P} f_{T, 1}+f_{T, 2}\right) s_{W}^{2}+\left(4 f_{T, 5}+f_{T, 6}+X f_{T, 6}+f_{T, 7}\right) c_{W}^{2}\right) /(2 \sqrt{2}) \\
\left(\left(4 f_{T, 0}+f_{T, 2}\right) s_{W}^{2}+\left(4 f_{T, 5}+f_{T, 7}\right) c_{W}^{2}\right) /(2 \sqrt{2})\end{array}$ \\
\hline$Z Z \rightarrow Z Z$ & $\begin{array}{l}+--+ \\
+-+- \\
++-- \\
++++\end{array}$ & $\begin{array}{l}X_{M}^{2}\left(\left(8 f_{T, 5}+8 f_{T, 6}+6 f_{T, 7}\right) c_{W}^{2}+\left(4 f_{T, 0}+4 f_{T, 1}+3 f_{T, 2}-8 f_{T, 5}-8 f_{T, 6}-6 f_{T, 7}\right) c_{W}^{4}+4\left(4 f_{T, 8}+3 f_{T, 9}\right) s_{W}^{4}\right) / 16 \\
X_{P}^{2}\left(\left(8 f_{T, 5}+8 f_{T, 6}+6 f_{T, 7}\right) c_{W}^{2}+\left(4 f_{T, 0}+4 f_{T, 1}+3 f_{T, 2}-8 f_{T, 5}-8 f_{T, 6}-6 f_{T, 7}\right) c_{W}^{4}+4\left(4 f_{T, 8}+3 f_{T, 9}\right) s_{W}^{4}\right) / 16 \\
(3+X)\left(2\left(4 f_{T, 5}+4 f_{T, 6}+f_{T, 7}\right) c_{W}^{2}+\left(4 f_{T, 0}+4 f_{T, 1}+f_{T, 2}-8 f_{T, 5}-8 f_{T, 6}-2 f_{T, 7}\right) c_{W}^{4}+4\left(4 f_{T, 8}+f_{T, 9}\right) s_{W}^{4}\right) / 8 \\
\left.\quad\left(\left(8 f_{T, 5}+8 f_{T, 6}+6 f_{T, 7}\right)\right) c_{W}^{2}+\left(4 f_{T, 0}+4 f_{T, 1}+3 f_{T, 2}-8 f_{T, 5}-8 f_{T, 6}-6 f_{T, 7}\right)\right) c_{W}^{4}+4\left(4 f_{T, 8}+3 f_{T, 9}\right) s_{W}^{4} / 4\end{array}$ \\
\hline$Z Z \rightarrow Z \gamma$ & $\begin{array}{l}+--+ \\
+-+- \\
++-- \\
++++\end{array}$ & $\begin{array}{l}X_{M}^{2}\left(\left(4 f_{T, 5}+4 f_{T, 6}+3 f_{T, 7}-16 f_{T, 8}-12 f_{T, 9}\right) s_{W}^{2}+\left(4 f_{T, 0}+4 f_{T, 1}+3 f_{T, 2}\right) c_{W}^{2}\right) s_{2 W} /(16 \sqrt{2}) \\
X_{P}^{2}\left(\left(4 f_{T, 5}+4 f_{T, 6}+3 f_{T, 7}-16 f_{T, 8}-12 f_{T, 9}\right) s_{W}^{2}+\left(4 f_{T, 0}+4 f_{T, 1}+3 f_{T, 2}\right) c_{W}^{2}\right) s_{2 W} /(16 \sqrt{2}) \\
(3+X)\left(\left(4 f_{T, 5}+4 f_{T, 6}+f_{T, 7}-16 f_{T, 8}-4 f_{T, 9}\right) s_{W}^{2}+\left(4 f_{T, 0}+4 f_{T, 1}+f_{T, 2}\right) c_{W}^{2}\right) s_{2 W} /(8 \sqrt{2}) \\
\quad\left(\left(4 f_{T, 5}+4 f_{T, 6}+3 f_{T, 7}-16 f_{T, 8}-12 f_{T, 9}\right) s_{W}^{2}+\left(4 f_{T, 0}+4 f_{T, 1}+3 f_{T, 2}\right) c_{W}^{2}\right) s_{2 W} /(4 \sqrt{2})\end{array}$ \\
\hline$Z Z \rightarrow \gamma \gamma$ & $\begin{array}{l}+--+ \\
+-+- \\
++-- \\
++++\end{array}$ & $\begin{array}{c}-X_{M}^{2}\left(\left(-4 f_{T, 0}-4 f_{T, 1}-3 f_{T, 2}+8 f_{T, 5}-2 f_{T, 7}-16 f_{T, 8}-12 f_{T, 9}\right) s_{2 W}^{2}-4\left(2 f_{T, 6}+f_{T, 7}\right) c_{2 W}^{2}\right) / 64 \\
-X_{P}^{2}\left(\left(-4 f_{T, 0}-4 f_{T, 1}-3 f_{T, 2}+8 f_{T, 5}-2 f_{T, 7}-16 f_{T, 8}-12 f_{T, 9}\right) s_{2 W}^{2}-4\left(2 f_{T, 6}+f_{T, 7}\right) c_{2 W}^{2}\right) / 64 \\
\left.8\left(4 f_{T, 5}+X_{P} f_{T, 6}+f_{T, 7}\right)+\left(4(3+X)\left(f_{T, 0}+f_{T, 1}-2\left(f_{T, 5}+f_{T, 6}-2 f_{T, 8}\right)\right)+\left(3+X^{2}\right)\left(f_{T, 2}-2 f_{T, 7}+4 f_{T, 9}\right)\right) s_{2 W}^{2}\right) / 32 \\
\left(\left(4 f_{T, 0}+4 f_{T, 1}+3 f_{T, 2}+16 f_{T, 5}-8 f_{T, 6}+16 f_{T, 8}+12 f_{T, 9}\right) s_{2 W}^{2}+4\left(4 f_{T, 5}+f_{T, 7}\right) c_{2 W}^{2}\right) / 16\end{array}$ \\
\hline$Z \gamma \rightarrow Z \gamma$ & $\begin{array}{l}+--+ \\
+-+- \\
++-- \\
++++\end{array}$ & $\begin{array}{c}-X_{M}^{2}\left(\left(-4 f_{T, 0}-4 f_{T, 1}-3 f_{T, 2}-8 f_{T, 5}+2 f_{T, 7}+8 f_{T, 6}-16 f_{T, 8}-12 f_{T, 9}\right) s_{2 W}^{2}-4\left(4 f_{T, 5}+f_{T, 7}\right) c_{2 W}^{2}\right) / 32 \\
-X_{P}^{2}\left(\left(-4 f_{T, 0}-4 f_{T, 1}-3 f_{T, 2}+8 f_{T, 5}+2 f_{T, 7}-16 f_{T, 8}-12 f_{T, 9}\right) s_{2 W}^{2}-4\left(2 f_{T, 6}+f_{T, 7}\right) c_{2 W}^{2}\right) / 32 \\
\left(2\left(2(5+3 X) f_{T, 6}+\left(4 f_{T, 5}+f_{T, 7}\right) X_{M}^{2}\right)+\left(2(3+X)\left(f_{T, 0}+f_{T, 1}-2\left(f_{T, 5}+f_{T, 6}-2 f_{T, 8}\right)\right)\right.\right. \\
\left.\left.\quad+\left(3+X^{2}\right)\left(2 f_{T, 0}+2 f_{T, 1}+f_{T, 2}-4 f_{T, 5}-4 f_{T, 6}-2 f_{T, 7}+8 f_{T, 8}+4 f_{T, 9}\right)\right) s_{2 W}^{2}\right) / 16 \\
\left(\left(4 f_{T, 0}+4 f_{T, 1}+3 f_{T, 2}-8 f_{T, 5}-2 f_{T, 7}+16 f_{T, 8}+12 f_{T, 9}\right) s_{2 W}^{2}+4\left(2 f_{T, 6}+f_{T, 7}\right) c_{2 W}^{2}\right) / 8\end{array}$ \\
\hline$Z \gamma \rightarrow \gamma \gamma$ & $\begin{array}{l}+--+ \\
+-+- \\
++-- \\
++++\end{array}$ & $\begin{array}{l}-X_{M}^{2}\left(\left(-4 f_{T, 0}-4 f_{T, 1}-3 f_{T, 2}+4 f_{T, 5}+4 f_{T, 6}+3 f_{T, 7}\right) s_{W}^{2}+\left(16 f_{T, 8}+12 f_{T, 9}\right) c_{W}^{2}\right) s_{2 W} /(16 \sqrt{2}) \\
\left.-X_{P}^{2}\left(\left(-4 f_{T, 0}-4 f_{T, 1}-3 f_{T, 2}+4 f_{T, 5}+4 f_{T, 6}+3 f_{T, 7}\right) s_{W}^{2}+\left(16 f_{T, 8}+12 f_{T, 9}\right) c_{W}^{2}\right) s_{2 W}\right) /(16 \sqrt{2}) \\
\left.-(3+X)\left(\left(-4 f_{T, 0}-4 f_{T, 1}-f_{T, 2}+4 f_{T, 5}+4 f_{T, 6}+f_{T, 7}\right) s_{W}^{2}+\left(16 f_{T, 8}+4 f_{T, 9}\right) c_{W}^{2}\right) s_{2 W}\right) /(8 \sqrt{2}) \\
\left.-\left(\left(-4 f_{T, 0}-4 f_{T, 1}-3 f_{T, 2}+4 f_{T, 5}+4 f_{T, 6}+3 f_{T, 7}\right) s_{W}^{2}+\left(16 f_{T, 8}+12 f_{T, 9}\right) c_{W}^{2}\right) s_{2 W}\right) /(4 \sqrt{2})\end{array}$ \\
\hline$\gamma \gamma \rightarrow \gamma \gamma$ & $\begin{array}{l}+--+ \\
+-+- \\
++-- \\
++++\end{array}$ & $\begin{array}{c}-X_{M}^{2}\left(-2\left(4 f_{T, 5}+4 f_{T, 6}+3 f_{T, 7}\right) c_{W}^{2}+2\left(4 f_{T, 5}+4 f_{T, 6}+3 f_{T, 7}-8 f_{T, 8}-6 f_{T, 9}\right) c_{W}^{4}-\left(4 f_{T, 0}+4 f_{T, 1}+3 f_{T, 2}\right) s_{W}^{4}\right) / 16 \\
-X_{P}^{2}\left(-2\left(4 f_{T, 5}+4 f_{T, 6}+3 f_{T, 7}\right) c_{W}^{2}+2\left(4 f_{T, 5}+4 f_{T, 6}+3 f_{T, 7}-8 f_{T, 8}-6 f_{T, 9}\right) c_{W}^{4}-\left(4 f_{T, 0}+4 f_{T, 1}+3 f_{T, 2}\right) s_{W}^{4}\right) / 16 \\
-(3+X)\left(-2\left(4 f_{T, 5}+4 f_{T, 6}+f_{T, 7}\right) c_{W}^{2}+2\left(4 f_{T, 5}+4 f_{T, 6}+f_{T, 7}-8 f_{T, 8}-2 f_{T, 9}\right) c_{W}^{4}-\left(4 f_{T, 0}+4 f_{T, 1}+f_{T, 2}\right) s_{W}^{4}\right) / 8 \\
\left.\quad\left(4 f_{T, 5}+4 f_{T, 6}+3 f_{T, 7}\right)\right) c_{W}^{2}+\left(-4 f_{T, 5}-4 f_{T, 6}-3 f_{T, 7}+8 f_{T, 8}+6 f_{T, 9}\right) c_{W}^{4}+\left(\left(8 f_{T, 0}+8 f_{T, 1}+6 f_{T, 2}\right) s_{W}^{4}\right) / 8\end{array}$ \\
\hline
\end{tabular}

TABLE VIII. Same as Table V but for the operators in the nonlinear representation of the electroweak symmetry [Eqs. (8) and (9)].

\begin{tabular}{lr}
\hline \hline & $\times g^{4} \frac{s^{2}}{M_{W}^{4}}$ \\
\hline$W^{+} W^{+} \rightarrow W^{+} W^{+}$ & $\left((5+X) f_{P, 11}+2(1+X) f_{P, 6}\right) / 16$ \\
$W^{+} Z \rightarrow W^{+} Z$ & $\left((5+3 X) f_{P, 11}-2(-1+X) f_{P, 23}+5 f_{P, 24}+3 X f_{P, 24}+2 f_{P, 6}-2 X f_{P, 6}\right) / 16$ \\
$W^{+} W^{-} \rightarrow W^{+} W^{-}$ & $\left((7+5 X) f_{P, 11}-2(-5+X) f_{P, 6}\right) / 16$ \\
$W^{+} W^{-} \rightarrow Z Z$ & $\left(f_{P, 11}+X f_{P, 11}+4 f_{P, 23}+f_{P, 24}+X f_{P, 24}+4 f_{P, 6}\right) /(8 \sqrt{2})$ \\
$Z Z \rightarrow Z Z$ & $\left((3+X)\left(f_{P, 11}+2 f_{P, 23}+2 f_{P, 24}+4 f_{P, 26}+f_{P, 6}\right)\right) / 8$ \\
\hline
\end{tabular}


[1] I. Brivio, T. Corbett, O. J. P. Éboli, M. B. Gavela, J. Gonzalez-Fraile, M. C. Gonzalez-Garcia, L. Merlo, and S. Rigolin, J. High Energy Phys. 03 (2014) 024.

[2] G. Belanger and F. Boudjema, Phys. Lett. B 288, 201 (1992).

[3] G. Belanger and F. Boudjema, Phys. Lett. B 288, 210 (1992).

[4] O. J. P. Eboli, M. C. Gonzalez-Garcia, S. M. Lietti, and S. F. Novaes, Phys. Rev. D 63, 075008 (2001).

[5] O. J. P. Eboli, M. C. Gonzalez-Garcia, and S. M. Lietti, Phys. Rev. D 69, 095005 (2004).

[6] S. Chatrchyan et al. (CMS Collaboration), J. High Energy Phys. 07 (2013) 116.

[7] S. Chatrchyan et al. (CMS Collaboration), Phys. Rev. D 90, 032008 (2014).

[8] G. Aad et al. (ATLAS Collaboration), Phys. Rev. Lett. 115, 031802 (2015).

[9] V. Khachatryan et al. (CMS Collaboration), J. High Energy Phys. 08 (2016) 119.

[10] V. Khachatryan et al. (CMS Collaboration), J. High Energy Phys. 06 (2017) 106.

[11] M. Aaboud et al. (ATLAS Collaboration), Phys. Rev. D 96, 012007 (2017).

[12] V. Khachatryan et al. (CMS Collaboration), Phys. Lett. B 770, 380 (2017).

[13] M. Aaboud et al. (ATLAS Collaboration), Eur. Phys. J. C 77, 646 (2017).

[14] A. M. Sirunyan et al. (CMS Collaboration), J. High Energy Phys. 10 (2017) 072.

[15] A. M. Sirunyan et al. (CMS Collaboration), Phys. Lett. B 774, 682 (2017).

[16] A. M. Sirunyan et al. (CMS Collaboration), Phys. Lett. B 798, 134985 (2019).

[17] A. M. Sirunyan et al. (CMS Collaboration), arXiv: 2002.09902.

[18] O. J. P. Éboli, M. C. Gonzalez-Garcia, and J. K. Mizukoshi, Phys. Rev. D 74, 073005 (2006).

[19] R. Alonso, M. B. Gavela, L. Merlo, S. Rigolin, and J. Yepes, Phys. Lett. B 722, 330 (2013); 726, 926(E) (2013).

[20] I. Brivio, O. J. P. Éboli, M. B. Gavela, M. C. GonzálezGarcia, L. Merlo, and S. Rigolin, J. High Energy Phys. 12 (2014) 004.

[21] J. M. Cornwall, D. N. Levin, and G. Tiktopoulos, Phys. Rev. D 10, 1145 (1974); 11, 972(E) (1975).
[22] E. Boos, H. J. He, W. Kilian, A. Pukhov, C. P. Yuan, and P. M. Zerwas, Phys. Rev. D 57, 1553 (1998).

[23] Y.-C. Guo, Y.-Y. Wang, and J.-C. Yang, arXiv:1912.10686.

[24] Y.-C. Guo, Y.-Y. Wang, and J.-C. Yang, arXiv:2002.03326.

[25] K. Arnold et al., Comput. Phys. Commun. 180, 1661 (2009).

[26] J. Kalinowski, P. Kozow, S. Pokorski, J. Rosiek, M. Szleper, and S. Tkaczyk, Eur. Phys. J. C 78, 403 (2018).

[27] P. Kozow, L. Merlo, S. Pokorski, and M. Szleper, J. High Energy Phys. 07 (2019) 021.

[28] G. Chaudhary, J. Kalinowski, M. Kaur, P. Kozow, K. Sandeep, M. Szleper, and S. Tkaczyk, Eur. Phys. J. C 80, 181 (2020).

[29] C. Garcia-Garcia, M. Herrero, and R. A. Morales, Phys. Rev. D 100, 096003 (2019).

[30] W. Buchmuller and D. Wyler, Nucl. Phys. B268, 621 (1986).

[31] C. N. Leung, S. T. Love, and S. Rao, Z. Phys. C 31, 433 (1986).

[32] A. De Rujula, M. B. Gavela, P. Hernandez, and E. Masso, Nucl. Phys. B384, 3 (1992).

[33] K. Hagiwara, S. Ishihara, R. Szalapski, and D. Zeppenfeld, Phys. Rev. D 48, 2182 (1993).

[34] M. C. Gonzalez-Garcia, Int. J. Mod. Phys. A 14, 3121 (1999).

[35] B. Grzadkowski, M. Iskrzynski, M. Misiak, and J. Rosiek, J. High Energy Phys. 10 (2010) 085.

[36] G. Passarino, Nucl. Phys. B868, 416 (2013).

[37] O. J. P. Éboli and M. C. Gonzalez-Garcia, Phys. Rev. D 93, 093013 (2016).

[38] S. Weinberg, Physica (Amsterdam) 96A, 327 (1979).

[39] F. Feruglio, Int. J. Mod. Phys. A 08, 4937 (1993).

[40] T. Appelquist and C. W. Bernard, Phys. Rev. D 22, 200 (1980).

[41] A. C. Longhitano, Nucl. Phys. B188, 118 (1981).

[42] M. Jacob and G. C. Wick, Ann. Phys. (N.Y.) 7, 404 (1959); 281, 774 (2000).

[43] H.-L. Li, Z. Ren, J. Shu, M.-L. Xiao, J.-H. Yu, and Y.-H. Zheng, arXiv:2005.00008.

[44] C. W. Murphy, arXiv:2005.00059.

[45] C. Csaki, C. Grojean, H. Murayama, L. Pilo, and J. Terning, Phys. Rev. D 69, 055006 (2004).

[46] T. Corbett, O. J. P. Éboli, and M. C. Gonzalez-Garcia, Phys. Rev. D 91, 035014 (2015). 